BRX TH-6305

CALT-TH 2016-006

\title{
Massive to gauge field reduction and gravitational wave zone information
}

\author{
S. Deser \\ Walter Burke Institute for Theoretical Physics, \\ California Institute of Technology, Pasadena, CA 91125; \\ Physics Department, Brandeis University, Waltham, MA 02454 \\ deser@brandeis.edu
}

\begin{abstract}
We show explicitly that massive, Abelian, vector - just like (properly defined) massive tensor - fields limit smoothly to their massless, gauge, versions: they emit only maximal helicity radiation and mediate Coulomb/"special relativistic Newtonian", forces between their (conserved) sources. Our main motivation, though, is to show that LIGO's gravitational wave detection probably cannot directly rule out very long-range gravity: Even though the waves were emitted in a strong field regime, their being detected in the weak field wave zone means the above equivalences apply. There remains the - not unlikely - possibility that no strong field generation of radiation in massive models can reproduce the observed ring-down patterns.

Separately, the smooth linear limiting behaviors show that the discontinuity lies not in the mass alone, but rather in Abelian versus non-Abelian - Yang-Mills and General Relativity regimes, whose respective massive versions are known to be non-physical.
\end{abstract}

\section{Introduction}

Some six decades ago, Bass and Schrödinger [1] examined massive vector, Proca, theory in an impassioned (the prose is pure Schrödinger) attempt to show that Maxwell electrodynamics is not an isolated model, but the smooth endpoint of a continuum of massive ones. Their results were not entirely complete, so one part of our work is to confirm them in full generality. However, this is for us just the introduction to the same question for general relativity's (GR) linearized spin 2, tensor field: is it also a smooth endpoint of the massive Fierz-Pauli (FP) model? We will indeed exhibit continuity 1 , then discuss its relevance to the recent spectacular LIGO observation of gravitational radiation in the weak field regime [2] and its full nonlinear strong field origins.

Of course we now know that the authors of [1] labored in an Abelian paradise and that both Yang-Mills and massive gravity nonlinear extensions are profoundly DIS-continuous in fundamental

\footnotetext{
${ }^{1}$ The sources of the respective fields must be conserved to preserve continuity. [One obvious symptom that non-conserved currents are problematic as $m \rightarrow 0$ is that $\partial^{\mu} A_{\mu} \sim m^{-2} \partial_{\mu} j^{\mu}$.] For spin one, this is a reasonable requirement on electric currents, but not for (even linearized ) spin 2, which is known to be inconsistent unless the sources become "slightly" covariantly conserved - and of course the field de-linearized as well.
} 
ways [3] in this limit, let alone the fact that even the Abelian spin 2 limit is only continuous in an (A)dS context [4]. However, it is still useful to settle the Abelian question for spin 2, to decide whether LIGO's detection of gravitational radiation amplitudes can rule out massive models observationally. We will find that at least the direct possibility is excluded 2 because the radiation arriving here, however strong its sources, is in a weak field, wave zone, regime [5], and thus still subject to the above Abelian continuity. Fortunately, there remains the, physically not unlikely, if slightly more indirect, possibility that massive gravity's strong field regime cannot generate waves with the observed ring-down amplitudes, though this is a very difficult calculation. Instead, the observed profile was accurately calculated long ago for GR in [6].

\section{Proca limits}

We want to show that the third, helicity 0, massive vector degree of freedom essentially decouples from sources and ceases to radiate as the mass vanishes; to this end, we use the ADM " $3+1$ " and orthogonal 3-space decompositions for spatial vectors and tensors [7], but keep second order form. Our signature is mostly plus. The Proca action, stress tensor and energy are

$$
\begin{aligned}
I_{m}[A] & =\int d^{4} x\left[-1 / 4 F_{\mu \nu}^{2}-1 / 2 m^{2} A_{\mu}^{2}+A_{\mu} j^{\mu}\right] \\
& =I_{m}\left[A_{i}^{T}\right]+I_{m}\left[A_{i}^{L}, A_{0}\right] \\
& \equiv 1 / 2 \int d^{4} x\left[\left(\dot{A}_{i}^{T}\right)^{2}-\left(A_{i}^{T}\right)^{2}\left(m^{2}-\nabla^{2}\right) A_{i}^{T}+2 A_{i}^{T} j_{i}^{T}\right] \\
& +1 / 2 \int d^{4} x\left[\left(\dot{A}_{i}^{L}\right)^{2}-m^{2}\left(\left(A_{i}^{L}\right)^{2}-A_{0}^{2}\right)+\left(\nabla A_{0}\right)^{2}-2 \dot{A}_{i}^{L} \partial_{i} A_{0}+2 A_{i}^{L} j_{i}^{L}+2 A_{0} j^{0}\right] \\
T_{\mu \nu} & =F_{\mu \alpha} F_{\nu}^{\alpha}-1 / 4 \eta_{\mu \nu} F_{\alpha \beta}^{2}+m^{2}\left(A_{\mu} A_{\nu}-1 / 2 \eta_{\mu \nu} A_{\alpha}^{2}\right) \\
E_{m} & =\int d^{3} x T_{00}=1 / 2 \int d^{3} x\left[\left(\partial_{0} A_{i}-\partial_{i} A_{0}\right)^{2}+m^{2}\left(A_{i}^{2}+A_{0}^{2}\right)\right] .
\end{aligned}
$$

This being a linear system, it is easy to separate the helicity 1 and 0 components by decomposing the 3 -vector fields and sources. The latter are taken to be external and conserved:

$$
\begin{aligned}
A_{i} & =A_{i}^{T}+A_{i}^{L}, \quad \nabla \cdot \mathbf{A}^{T}=0=\nabla \times \mathbf{A}^{L}, \quad \int d^{3} x \mathbf{V}^{T} \cdot \mathbf{W}^{L}=0, \quad A_{i}^{L} \equiv \frac{\partial_{i}}{\sqrt{-\nabla^{2}}} a(4) \\
\partial_{\mu} j^{\mu} & =0 \equiv \nabla \cdot \mathbf{j}+\partial_{0} j^{0} \equiv \sqrt{-\nabla^{2}} j+\dot{\rho} .
\end{aligned}
$$

The $A_{i}^{T}$ helicity 1 action manifestly decouples from the rest. Its only $m$-dependence being through the massive, rather than massless, propagator $\left(\square-m^{2}\right)^{-1}$, the limit indeed smoothly reduces to the Maxwell action in all its radiative aspects, namely dipole waves emitted by the transverse currents, and their retarded interactions mediated by the transverse "photon". The Coulomb term will emerge presently, as the (only) survivor of the helicity 0 sector; this is a purely bookkeeping

\footnotetext{
${ }^{2}$ While LIGO does place upper limits on the "graviton's" mass (or more properly, lower limits on its range), they are weaker than those from cosmological scale arguments.
} 
choice of where we placed $A_{0}$. The remaining, longitudinal action is, from (1),

$$
\begin{aligned}
I[a] & =1 / 2 \int d^{4} x\left[\dot{a}^{2}-m^{2} a^{2}+2 a j\right]-1 / 2 \int d^{4} x\left[\rho Y \rho-2 \rho Y \sqrt{-\nabla^{2}} \dot{a}+\dot{a} Y\left(-\nabla^{2}\right) a\right] ; \\
Y & \equiv\left(m^{2}-\nabla^{2}\right)^{-1},
\end{aligned}
$$

after completing squares in the $A_{0}^{2}+A_{0}$ constraint sector of (1), to remove its $A_{0}$ dependence. It now remains to establish the consequences of this helicity zero action; this requires repeated use of current conservation, (5). We expect, following [1], the massless limit to be smooth and yield a free helicity 0 excitation, together with the standard instantaneous Coulomb interaction. [Note in this connection that this otherwise inert excitation remains (unavoidably) coupled to gravity, so that if it had been copiously produced at the Big Bang - if not by radiating charges - it would forever exist as truly dark matter.] We merely sketch the process: Redefining a in terms of a new variable $\mathrm{S}$,

$$
a=\sqrt{1-m^{-2} \nabla^{2}} S
$$

eventually yields

$$
I(S)=1 / 2 \int d^{4} x\left[S \square S+1 / 2 \int d^{4} x \rho \nabla^{-2} \rho+O(m)\right.
$$

as expected 3 .

The net result then is that Proca indeed limits smoothly to Maxwell plus a free helicity 0 mode that only couples to gravity. The above interaction results can also be read off from the effective current-current action,

$$
I_{m}(j)=1 / 2 \int d^{4} x j^{\mu} \Delta j_{\mu}, \quad \Delta \equiv\left(\square-m^{2}\right)^{-1}
$$

The $j^{T}$ parts indeed couple with retardation (and of course $\Delta$ reduces smoothly to the massless propagator $D$ ), while the, ostensibly also retarded, remainder reduces straightforwardly, upon using conservation, precisely to the Coulomb form in (8).

\section{$3 \quad$ Massive spin 2}

We now turn to our main interest - spin 2 - including the $m \rightarrow 0$ reduction of FP to linearized GR. Rather than subject the reader to dissection of the full action à la spin 1 above - its free part is already messy enough, and has already been separated into its helicity sectors [8], we concentrate on the source-source, $T_{\mu \nu}-T_{\mu \nu}$ interactions, which is of course equivalent to reading off the propagator. The free, kinematical part consists of the five, helicity $( \pm 2, \pm 1,0)$ sectors, the 3 lower "longitudinal" ones again non-interacting, while the helicity 2 propagates according to $\Delta$, i.e., with $D$ on the light cone in the limit. The T-T action is trivial to obtain because the numerators of the FP (or gravity) propagator involve projectors whose $m^{-2} \partial_{\mu} \partial_{\nu}$ term vanish when acting on the conserved $T_{\mu \nu}$. We first encounter here the ancient vDVZ [9] "paradox", that the the respective numerators differ by

\footnotetext{
${ }^{3}$ While the redefinition (7) is singular at $m=0$, this simply reflects the unavoidable discontinuity of degree of freedom count between Proca and Maxwell. Indeed, the only way to understand the amount of radiation of these longitudinal waves is to put their action into normalized form (8), that is with a normal free field part.
} 
a constant, even in the massless limit:

$$
I[m \rightarrow 0 ; m=0]=1 / 2 \int d^{4} x\left[T_{\mu \nu} D T^{\mu \nu}-(1 / 3 ; 1 / 2) \operatorname{tr} T D \operatorname{tr} T\right] .
$$

[An easy way to understand why there is a discontinuity is that the sum of the trace and double divergence of the massive field equations yields the discontinuous field-current identity [10] tr $h_{\nu}=$ $m^{-2} \operatorname{tr} T_{\mu \nu}$.] Fortunately, it was later discovered [4] that the discontinuity could be avoided by using the old axiom that whatever is not prohibited is compulsory: in presence of a non-vanishing cosmological constant, the massive propagator's second term magically reverts to $1 / 2$ from $1 / 3$, while the massless one stays at $1 / 2$. [One can take the Minkowski, $\Lambda=0$, limit afterwards if desired.] Thus, we may indeed analyze (10) with the $1 / 2$ value (the transition from $\Delta$ to $D$ propagators is of course again smooth). Indeed, this requires no effort: Linearized gravity action's consequences are well enough known! They automatically imply the same miracle as in the Proca case: the radiation from the lower helicity modes is suppressed as $m$ vanishes. More specifically, exactly the procedure detailed above for spin 1 - decomposition of the fields and sources into their irreducible $3+1$ parts, together with use of conservation of $T_{\mu \nu}$ - leads, by straightforward but tedious computation to retarded helicity 2, quadrupole $\int T_{i j}^{T T} D T_{i j}^{T T}$, transverse-traceless $\left(\partial^{j} T_{i j}^{T T}=0=T_{i i}^{T T}\right)$, interaction plus "Newtonian" instantaneous ones among the other (unconstrained by conservation) components of $T_{\mu \nu}$, just as in linearized GR .

\section{Wave zone}

Before the above linearized conclusions can be applied to the LIGO observations, we must face the fact that emission took place in a (very) strong field regime, where linearization does not apply. While a simple problem in the Abelian case, as in textbook Maxwell theory, setting up the concordance between strong interior emission and distant observation becomes a major problem in GR. Fortunately, it has been solved, in a lengthy technical paper [5], which dealt, in a (physically, at least) rigorous way, with the transition to asymptotic falloff of the strong field radiation excitations, unclouded by coordinate-and gravitational interaction-dependent issues. The upshot is that there is a well-defined spatial domain where physical modes behave sufficiently close to plane waves subject to linearized GR, hence with no memory of the actual interior emission's details, though of course providing enough details to enable one to reconstruct the black hole merger as a natural emission candidate. While the same analysis has never been attempted for the massive models, it seems safe to say that the conclusions about its wave zone should not differ substantially: the mass terms are relatively easy to tame and there are fewer "coordinate wave" complications, given the loss of coordinate invariance here in favor of a fixed background. On the other hand, deciding if here too there is a corresponding strong field emission scenario is not an obvious matter!

\section{Conclusion}

The conclusions we can draw are that massive gravity waves also have a wave zone effectively decoupled form the interior, and subject to (linear) FP propagation; that is, for very small $m$, 
only quadrupole waves emerge and are indistinguishable from the mass $=0$ GR case. LIGO's observation's inability to distinguish directly between GR and its massive extension is based on two facts: the similarity of the two models' wave-zone properties and of their linearized versions governing that region. However, the violent black hole merger process emitting the waves in GR might require a very different origin in the massive models, where even what black holes and their mergers mean has never been studied sufficiently. Indeed, there might simply be no strong field event that would generate the ring-down profile inferred from LIGO. So it is possible that massive gravity has already been excluded, but if so, through differences in the radiating interiors' dynamics rather than from $m$-dependent pure wave zone discrepancies.

\section{Acknowledgements.}

This work was supported by grants NSF PHY-1266107 and DOE \# desc0011632. I thank J. Franklin for compositional dexterity.

\section{References}

[1] L. Bass and E. Schrödinger, Proc. R. Soc. Lond. A 232, 1 (1955).

[2] The LIGO Scientific and Virgo Collaborations, Phys. Rev. Lett.116, 061102 (2016), grqc/1602.03837.

[3] For Yang-Mills: D.G. Boulware, Annals Phys. 56, 140(1970). For massive gravity: S. Deser, A. Waldron, G. Zahariade, Phys.Lett. B749, 144(2015), hep-th 1504.02919; S. Deser, K. Izumi, Y.C. Ong, A. Waldron, Mod.Phys.Lett. A30 (2015)1540006, hep-th/1410.2289, and references therein.

[4] I.I. Kogan, S. Mouslopoulos and A. Papazoglou, Phys.Lett. B503 173 (2001), hep-th/0011138; M. Porrati Phys.Lett. B498 92(2001), hep-th/0011152 see also A. Higuchi, Nucl. Phys. B282 397(1987); ibid. B325, 745(1989).

[5] R. Arnowitt, S. Deser, C.W.Misner, Phys Rev 121,1556(1961).

[6] Alessandra Buonanno, Thibault Damour, Phys.Rev. D62 (2000) 064015, gr-qc/0001013.

[7] R. Arnowitt, S. Deser, C.W.Misner, in Gravitation: an introduction to current research, L. Witten, ed. (Wiley, New York, 1962); reprinted in Gen.Rel.Grav. 401997(2008), and gr-qc/0405109.

[8] M. Fierz, Helv. Phys. Acta 12,3 (1939); M. Fierz and W. Pauli, Proc. R. Soc. Lond A173 211(1939); S. Deser, Can.J.Phys. 93, 395 (2015), gr-qc/1407.7178.

[9] H. van Dam and M. Veltman, Nucl. Phys. B22 397(1970) ; V.I. Zakharov, JETP Lett. 12, $312(1970)$.

[10] S. Deser, A. Waldron, Phys. Rev. D 89, 027503 (2014), gr-qc/1310.2675 